\title{
Electromagnetic Oscillator Action as an Introduction to Discrete Physics
}

\author{
Milan Perkovac \\ College for Information Technologies, Zagreb, Croatia.
}

How to cite this paper: Milan Perkovac. (2020) Electromagnetic Oscillator Action as an Introduction to Discrete Physics. Journal of Applied Mathematics and Computation, 4(3), 64-82.

DOI: 10.26855 /jamc. 2020.09 .003

Received: June 23, 2020

Accepted: July 18, 2020

Published: August 11, 2020

*Corresponding author: Milan Perkovac, College for Information Technologies, Zagreb, Croatia.

Email: milan@drivesc.com

\begin{abstract}
The aim of this study is to define both the structural constant of all atoms $s_{0}$ and the action of $L C$ oscillator $A$, as a new concept. The methods of theoretical research are used, and its checking is based on previously measured data. Electromagnetic radiation, which we observe in an area outside of the atom, has its source in the atom. As a model of this source an $L C$ oscillator was investigated within the atom. It is determined that the energy of that $L C$ oscillator is proportional to its natural frequency. However, the proportionality factor $A$, which is analogous to Planck's $h$, is not constant, but decreases with the increase in this frequency. Periodic coincidence of two independent phenomena within an atom is condition of the stability of an atom. These two phenomena are, first, circulating the electron around the nucleus, and second, oscillating the electromagnetic energy in the atom. At the integer frequency ratio of these two phenomena, discretization of the atoms state occurs. The structural constant and its unified value is defined; $s_{0}=8.278691910$. All NIST Data, from Hydrogen, ${ }_{1} \mathrm{H}$, to Darmstadtium, ${ }_{110} \mathrm{Ds}, 110$ metrics, confirmed this value. This approach, besides the atomic shell, includes its nucleus. It is shown that with help of structural constant $s_{0}$, as well with help of the other five known constants $\left(c, \mu_{0}, e, m, m_{\mathrm{p}}\right)$, nine existing constants become redundant; i.e., fine structure constant $\alpha$, von Klitzing constant $R_{\mathrm{K}}$, Planck's $h$, ratio $e / h$, Josephson constant $K_{\mathrm{J}}$, Rydberg constant $R_{\infty}$, Bohr radius $a_{0}$, Bohr magneton $\mu_{\mathrm{B}}$, and nuclear magneton $\mu_{\mathrm{N}}$. All relevant physical quantities are also given in a form suitable for use in Discrete Physics. All relations in Discrete Physics are as clear as in Classical Physics.
\end{abstract}

\section{Keywords}

Discrete Physics, Discrete States of Atoms, Lecher Line, Maxwell's Equations, Structural Constant, Unit for Type of Substance

\section{Introduction}

At the very beginning, it should be noted that the theory presented here is based on Maxwell's electromagnetism and on relativity theory. Emission of radiation during acceleration, known as radiation reaction [1], or radiation caused by the mechanical oscillation of charge [2,3], are much less in relation to the radiation resulting from the assumed $L C$ oscillator in the atom. This $L C$ oscillator I use after I read Planck's review on the Bohr model of the atom [4]. Complaints about the collapse of atoms in such theories are eliminated through full access to the atom which includes emission and absorption of the radiation $[5,6]$, where it is possible to absorb and its own radiation emission. Electromagnetic radiation, which we observe in an area outside of the atom, has its source in the atom. As a model of this source an $L C$ oscillator was investigated within the atom. 


\section{The Atom as an $L C$ Oscillator}

The $L C$ oscillator is most commonly used to generate electromagnetic waves for practical use, for example radio, television or communication signals. However, it is theoretically possible to obtain any electromagnetic wave of resonant frequency $\omega=2 \pi f=1 / \sqrt{L C}$ using the $L C$ oscillator. Emitted electromagnetic energy $E_{\text {em }}$ of such oscillator can be calculated in several ways. One way is to integrate the entire energy of the emitted electromagnetic fields [7],

$$
E_{e m}=\frac{1}{2} \iiint(\boldsymbol{E} \cdot \boldsymbol{D}+\boldsymbol{H} \cdot \boldsymbol{B}) d x d y d z
$$

where $\boldsymbol{E}$ and $\boldsymbol{H}$ are electric and magnetic field respectively, $\boldsymbol{D}$ is electric displacement and $\boldsymbol{B}$ is magnetic induction.

Another way is to calculate the loss of energy of the $L C$ oscillator as a source of electromagnetic energy, with use the law of conservation of energy; this means that the energy emitted by $L C$ oscillator becomes the energy of the electromagnetic wave. We will consider the situation that the source of the electromagnetic wave is a structure of the substance that it consists of a central body with a charge $Q$, while around this body at a distance $r$ (orbit radius), with uniform speed $v$ in circular motion revolving the body with mass $m$ and with the charge $q$. Then it is $v / c=\beta$, and $c$ is the speed of light in vacuum. This moving body has an acceleration directed radially toward the center the circle, i.e., at right angel to the vector of velocity $\mathbf{v}$ of magnitude [8] $v^{2} / r$. Relativistic moment $\boldsymbol{p}$ of moving body, [8], is

$$
\boldsymbol{p}=\frac{m \mathbf{v}}{\sqrt{1-\beta^{2}}} .
$$

In the stationary state, when the variables are at their fixed amounts, the Newton second law of motion, [8], $\boldsymbol{F}=d \boldsymbol{p} / d t=d\left(m \mathbf{v} / \sqrt{1-\beta^{2}}\right) / d t=\left(m / \sqrt{1-\beta^{2}}\right)(d \mathbf{v} / d t)=\left(m / \sqrt{1-\beta^{2}}\right)\left(v^{2} / r\right)(\boldsymbol{r} / r)$ and Coulomb's the law of attraction, [8], $q Q / 4 \pi \varepsilon_{0} r^{2}$, are equalized; we take that $q$ and $Q$ have opposite sign and $q$ is negative:

$$
\frac{m v^{2}}{r \sqrt{1-\beta^{2}}}=-\frac{q Q}{4 \pi \varepsilon_{0} r^{2}}
$$

here $\varepsilon_{0}$ is electric constant. From Eq. (3) it follows (with $v / c=\beta$ ):

$$
r=-\frac{q Q}{4 \pi \varepsilon_{0} m c^{2}} \frac{\sqrt{1-\beta^{2}}}{\beta^{2}} .
$$

The kinetic energy of the moving body is [8]

$$
K=\frac{m c^{2}}{\sqrt{1-\beta^{2}}}-m c^{2},
$$

and its potential energy [8], using Eq. (4), is

$$
U=\frac{q Q}{4 \pi \varepsilon_{0} r}=q V_{U}=-\frac{m c^{2}}{\sqrt{1-\beta^{2}}} \beta^{2},
$$

here $V_{U}=Q /\left(4 \pi \varepsilon_{0} r\right)$ is electric potential [8], also known as the Coulomb potential.

The total mechanical energy $W$ of the observed system is the sum of previously determined kinetic energy, $K$, and the potential energy, $U[8]$ :

$$
W=K+U=-m c^{2}\left(1-\sqrt{1-\beta^{2}}\right) .
$$

To keep this system stationary (meaning it no longer emits or absorbs energy), it has by law of conservation of energy emitted exactly such a large amount of energy $\Delta W=W-W_{0}=-m c^{2}\left(\sqrt{1-\beta_{0}^{2}}-\sqrt{1-\beta^{2}}\right)=E_{e m}$, with the opposite 
$\operatorname{sign} \Delta W$ and $E_{e m}$; namely, the energy lost by the atom, $\Delta W$, gets to the emitted photon, $E_{e m}$. For simplicity, we will take that initial velocity is always zero, i.e., $\beta_{0}=0$. In this way, we calculated the amount of electromagnetic energy emitted,

$$
E_{e m}=-W=m c^{2}\left(1-\sqrt{1-\beta^{2}}\right)=-q V_{e m},
$$

where $V_{e m}$ is the potential difference (voltage) through which passes the body charged with charge $q$ to get the same energy as the electromagnetic energy $E_{e m}$ emitted (as we have said, the charge $q$ is negative; $q=-z e, z=1,2,3, \ldots, e$ is the elementary charge, while $Q=+Z e, \mathrm{Z}=1,2,3, \ldots)$.

From Eq. (8), $E_{e m}=m c^{2}\left(1-\sqrt{1-\beta^{2}}\right)$, we can express $\sqrt{1-\beta^{2}}=\left(1-E_{e m} / m c^{2}\right)$ and $\beta^{2}=2 E_{e m}\left(1-E_{e m} / 2 m c^{2}\right) / m c^{2}$, and we include these two in the Eq. (4); we get

$$
r=-\frac{q Q}{8 \pi \varepsilon_{0} E_{e m}} \frac{1-E_{e m} / m c^{2}}{1-E_{e m} / 2 m c^{2}}
$$

and from here

$$
E_{e m}=-\frac{1}{2} \frac{q Q}{4 \pi \varepsilon_{0} r} \frac{1-E_{e m} / m c^{2}}{1-E_{e m} / 2 m c^{2}} .
$$

With $E_{e m}=-q V_{e m}$ and $\varepsilon_{0}=1 / \mu_{0} c^{2}$, from Eq. (10) we get two solutions for $V_{e m}$, from infinite distance to the first orbit:

$$
V_{e m 1}=\frac{c^{2}\left(\mu_{0} q Q-8 m \pi r+\sqrt{\mu_{0}^{2} q^{2} Q^{2}+8^{2} m^{2} \pi^{2} r^{2}}\right)}{8 \pi q r},
$$

and from the first orbit to a distance equal to zero:

$$
V_{e m 2}=\frac{c^{2}\left(\mu_{0} q Q-8 m \pi r-\sqrt{\mu_{0}^{2} q^{2} Q^{2}+8^{2} m^{2} \pi^{2} r^{2}}\right)}{8 \pi q r},
$$

while from equation (9) a unique solution for $r$ arises:

$$
r=\left|\frac{\mu_{0} c^{2} Q}{8 \pi V_{e m}} \frac{1+\frac{q V_{e m}}{m c^{2}}}{1+\frac{q V_{e m}}{2 m c^{2}}}\right| .
$$

So, for example, with measured [9] $V_{e m}=13.59843449 \mathrm{~V},(q=-z e=-e ; Q=Z e=e)$, from Eq. (13) we calculate the radius of the first Hydrogen orbit, $r_{\mathrm{H}}=5.294526279 \times 10^{-11} \mathrm{~m}$, and with $V_{e m}=54.417765 \mathrm{~V}$, ( $q=-z e=-e ; Q=Z e=2 e)$, we get the radius of the first orbit of Helium, $r_{\mathrm{He}}=2.645988600 \times 10^{-11} \mathrm{~m}$, and with $V_{e m}=1362.19915 \mathrm{~V},(q=-z e=-e ; Q=Z e=10 e)$, we get the first orbit of Neon, $r_{\mathrm{Ne}}=5.278386334 \times 10^{-12} \mathrm{~m}$, while for Darmstadtium, $V_{e m}=204400 \mathrm{~V},(q=-z e=-e ; Q=Z e=110 e)$, the first orbit is $r_{\mathrm{Ds}}=2.905992468 \times 10^{-13}$ $\mathrm{m}$.

To create an electromagnetic wave, as stated, which comes from the observed structure, this structure in some way enables its broadcasting. Let's examine the existence of the simple harmonic $L C$ oscillator in that structure. The natural frequency $f$ of the $L C$ oscillator is [8].

$$
f=\omega / 2 \pi=1 /(2 \pi \sqrt{L C})
$$


The electromagnetic energy $E_{e m}$ in the observed structure, which can be an atom too, is the energy of the observed $L C$ oscillator [8].

$$
E_{e m}=\frac{1}{2} \frac{\Theta^{2}}{C}=\frac{1}{2} L I^{2}=\frac{1}{2} L \omega^{2} \Theta^{2} ;
$$

$\Theta$ is here the maximum charge on the condenser whose capacitance is $C$. The correlation between the frequency $f$ and the angular frequency $\omega$ is in Eq. (14), $\omega=2 \pi f=1 / \sqrt{L C}$, and from this last expression comes the inductance $L$ :

$$
L=1 /\left(\omega^{2} C\right)=1 /\left(4 \pi^{2} f^{2} C\right) \text {. }
$$

When we equate Eqs. $(10,15)$ then we get:

$$
-\frac{1}{2} \frac{q Q}{4 \pi \varepsilon_{0} r} \frac{1-E_{e m} / m c^{2}}{1-E_{e m} / 2 m c^{2}}=\frac{1}{2} \frac{\Theta^{2}}{C} .
$$

In only one Eq. (17) there are two unknowns, $C=x$ and $\Theta=y$. It would be useful to establish another independent equation. If that is not possible then we can try to solve this equation in another way. When the number of unknowns is greater than the number of equations then the solution is searched by Diophantine equations [10]. In order to solve the Eq. (17) we describe it in algebraic form:

$$
a x+b y^{2}=0
$$

there are $a$ and $b$ coefficients of Eq. (18):

$$
a=\frac{1}{4 \pi \varepsilon_{0} r} ; \quad b=\frac{1}{q Q \frac{1-E_{e m} / m c^{2}}{1-E_{e m} / 2 m c^{2}}} .
$$

Since coefficients $a$ and $b$ are not integers, as Diophantine equations require, the solution should be sought in another way. One set of solutions obviously is

$$
C=4 \pi \varepsilon_{0} r,
$$

and

$$
\Theta^{2}=-q Q \frac{1-E_{e m} / m c^{2}}{1-E_{e m} / 2 m c^{2}} .
$$

Eq. (17) will now be written in a different way. It is a path to show that electromagnetic energy in $L C$ oscillator always is proportional to its natural frequency $f$, Eq. (14). Namely, according to the Eq. (15) is:

$$
\begin{aligned}
& E_{e m}=\frac{1}{2} \frac{\Theta^{2}}{C}=\frac{1}{2} \frac{\pi}{\pi} \frac{\Theta^{2}}{\sqrt{C} \sqrt{C}} \frac{\sqrt{L}}{\sqrt{L}} \\
& =\pi \sqrt{\frac{L}{C}} \Theta^{2} \frac{1}{2 \pi \sqrt{L C}} \\
& =\pi \mathrm{Z}_{L C} \Theta^{2} f \\
& =\pi \sqrt{\frac{L}{C}}\left(-q Q \frac{1-E_{\mathrm{em}} / m c^{2}}{1-E_{\mathrm{em}} / 2 m c^{2}}\right) f=A f .
\end{aligned}
$$

Here $A$ is the action of the electromagnetic $L C$ oscillator; it is the quotient of the electromagnetic energy $E_{e m}$ to the natural frequency $f$ of the $L C$ oscillator:

$$
A=\frac{E_{e m}}{f}=-\pi \sqrt{\frac{L}{C}} q Q \frac{1-E_{\mathrm{em}} / m c^{2}}{1-E_{\mathrm{em}} / 2 m c^{2}} .
$$

We have the capacitance of $L C$ oscillator mathematically chosen in Eq. (20), i.e., $C=4 \pi \varepsilon_{0} r$. The $L C$ oscillator 
inductance $L$ is thereby also being chosen in accordance with Eq. (16), $L=1 /\left(4 \pi^{2} f^{2} C\right)$. So, the characteristic impedance of $L C$ oscillator, which impedance can be expressed as a $\gamma$ multiplier with vacuum impedance $Z_{0}=\sqrt{\mu_{0} / \varepsilon_{0}}=\mu_{0} c$, is

$$
\begin{aligned}
& \mathrm{Z}_{L C}=\sqrt{\frac{L}{C}}=\gamma Z_{0}=\gamma \mu_{0} c \\
& =\sqrt{\frac{1 /\left(4 \pi^{2} f^{2} C\right)}{C}} \\
& =\sqrt{\frac{1}{4 \pi^{2} f^{2} C^{2}}}=\frac{1}{2 \pi f C},
\end{aligned}
$$

whereby $\gamma=Z_{L C} / Z_{0}$ is the impedances ratio [5], and when we put the result from Eq. (24) in Eq. (23) we get:

$$
A=-\pi \frac{1}{2 \pi f C} q Q \frac{1-E_{e m} / m c^{2}}{1-E_{e m} / 2 m c^{2}} .
$$

Now we can include in Eq. (25) the amount of $C$ from Eq. (20):

$$
A=-\frac{1}{8 f \pi \varepsilon_{0} r} q Q \frac{1-E_{e m} / m c^{2}}{1-E_{e m} / 2 m c^{2}} .
$$

If we include now $r$, from Eq. (4) in Eq. (26), we get:

$$
\begin{aligned}
& A=-\frac{q Q \frac{1-E_{e m} / m c^{2}}{1-E_{e m} / 2 m c^{2}}}{8 f \pi \varepsilon_{0}\left(-\frac{q Q}{4 \pi \varepsilon_{0} m c^{2}} \frac{\sqrt{1-\beta^{2}}}{\beta^{2}}\right)} \\
& =\frac{m c^{2} \beta^{2}}{2 f \sqrt{1-\beta^{2}}} \frac{1-E_{e m} / m c^{2}}{1-E_{e m} / 2 m c^{2}} .
\end{aligned}
$$

Let's just note now one detail; namely, the Eq. (27) is not directly dependent on the charge. Later [after Eq. (47)] we will return to Eq. (27) and show that term $m c^{2} \beta^{2} /\left(2 f \sqrt{1-\beta^{2}}\right)$ is actually a constant.

\section{Lecher Line as Model of an $L C$ Atom Oscillator}

To clarify relationship between $\beta$ and $f$ in Eq. (27), in term $m c^{2} \beta^{2} /\left(2 f \sqrt{1-\beta^{2}}\right)$, we will use a known source of electromagnetic energy, for example a Lecher transmission line (Figure 1), where the physical relations of the $L C$ oscillator are entirely clear. Specifically, we assume Maxwell's equations also apply to the atom. The equations of Lecher's lines are completely in line with Maxwell's equations. That is why Lecher's line in terms of electromagnetic oscillations can be used as part of the atomic model. Once we clarify these term $m c^{2} \beta^{2} /\left(2 f \sqrt{1-\beta^{2}}\right)$, after Eq. (47), then we will return to Eq. (27). This, in effect, means that we will use the Lecher line as a model of the oscillatory $L C$ oscillator in the atom. Note here that this could be some other transmission line. Lecher line has been chosen as a model because of its simplicity.

Lecher line is twin-lead transmission line consisting of a pair of ideal conductive nonmagnetic parallel wires of diameter $2 \rho$, separated by $\delta$ (we tag $\delta / \rho=\chi$ ), whose length is $\zeta$, situated in space with permittivity $\varepsilon_{0}$ and permea- 




a)

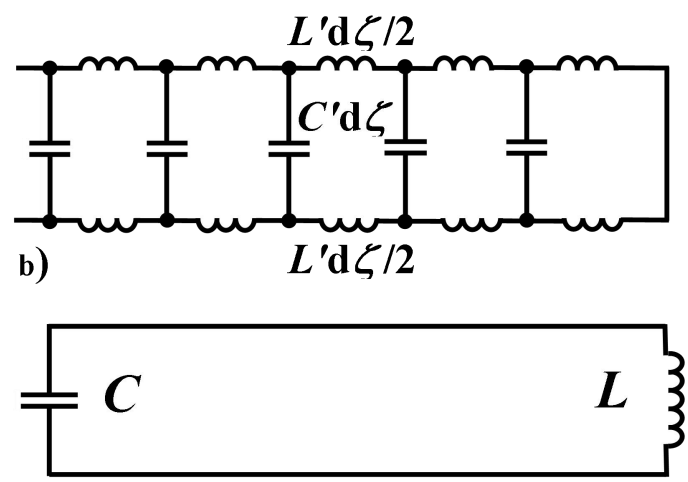

c)

Figure 1. a) A section of Lecher's line that is long $\zeta$; it is a twin-lead transmission line consisting of pair of ideal conductive nonmagnetic wires of diameter $2 \rho$, separated by $\delta$, situated in space with permittivity $\varepsilon_{0}$ and permeability $\mu_{0}$.

b) Lecher's line presented by an infinite number of extremely small uniformly distributed capacitors, with capacitance $C^{\prime} d \zeta$, and with equals such inductors, with inductance $L$ 'd $\zeta$.

c) All mentioned capacitors are collected at the open end of the line, denoted by $C$, and all mentioned inductors are collected on its short-circuited end, and denoted by $L$, resulting in an $L C$ circuit.

bility $\mu_{0}$. Let's repeat the procedure for the Lecher line starting with Eq. (24). Capacitance per unit length $C^{\prime}$ of Lecher line is [11].

$$
C^{\prime}=\pi \varepsilon_{0} / \ln \left(\chi / 2+\sqrt{(\chi / 2)^{2}-1}\right)
$$

and inductance per unit length $L^{\prime}$ of Lecher line is [11],

$$
L^{\prime}=\mu_{0}(\ln \chi+1 / 4) / \pi .
$$

So, the characteristic impedance of the Lecher line is

$$
\begin{aligned}
& Z_{L C}=\sqrt{\frac{L}{C}}=\sqrt{\frac{L^{\prime} \mathrm{d} \zeta}{C^{\prime} \mathrm{d} \zeta}}=\sqrt{\frac{L^{\prime}}{C^{\prime}}} \\
& =\sqrt{\frac{\mu_{0}}{\varepsilon_{0}}} \frac{\sqrt{\left[\ln \left(\frac{\chi}{2}+\sqrt{\left(\frac{\chi}{2}\right)^{2}-1}\right)\right]\left(\ln \chi+\frac{1}{4}\right)}}{\pi} \\
& =\sqrt{\frac{\mu_{0}}{\varepsilon_{0}}} \frac{\sigma(\chi)}{\pi}
\end{aligned}
$$

while

$$
\sigma(\chi)=\sqrt{\left[\ln \left(\frac{\chi}{2}+\sqrt{\left(\frac{\chi}{2}\right)^{2}-1}\right)\right]\left(\ln \chi+\frac{1}{4}\right)}
$$

is the structural coefficient of Lecher line. 
Take the segment of equation (22), $\Theta^{2} /(2 C)=\pi \mathrm{Z}_{L C} \Theta^{2} f$, and include in it Eq. (20) (i.e., $C=4 \pi \varepsilon_{0} r$ ), and Eq. (30) [i.e., $Z_{L C}=\sqrt{\mu_{0} / \varepsilon_{0}} \sigma(\chi) / \pi$ ], we get the natural frequency of the oscillatory circuit. This frequency depends only on the parameters of the oscillatory circuit and the properties of the space in which that circuit is located:

$$
f=\frac{1}{8 \pi \sqrt{\varepsilon_{0} \mu_{0}} \sigma(\chi) r} .
$$

\section{Structural Constant of all Atoms}

Let us notice precisely here that the natural frequency $f$ in Eq. (32) depends only on the properties of the space, i.e., $\varepsilon_{0}$, and $\mu_{0}$, and on the spatial parameters, i.e., $\chi$, in accordance with Eq. (31) and $r$. The Eqs. $(27,32)$ are not dependent on charges or any charge product. Thus in accordance with Eq. (22) neither the energy $E_{e m}=A f$, does not explicitly depend on the charge, because neither $A$ nor $f$ explicitly depend on the charge. However, if the radius $r$ from Eq. (9) is entered in equation (32) we get:

$$
f=-\frac{E_{e m}}{\sqrt{\mu_{0} / \varepsilon_{0}} \sigma(\chi) q Q} \frac{1-E_{e m} / 2 m c^{2}}{1-E_{e m} / m c^{2}} .
$$

Here, the charge appears as a variable in the form of product $q Q$. To avoid this, and realize the frequency $f$ independent on the charge as variables, should be taken to be worth $\sigma(\chi) q Q=-\sigma(\chi) z Z e^{2}=$ Const.1, actually, since $e^{2}$ is constant in itself, $-\sigma(\chi) z Z=$ Const. $2=s_{0}^{2}$, with, as stated above, $q=z(-e)=-z e$ and $Q=Z(+e)=Z e$, where elementary charge $e$ is used only as measure of the charge, but not as a variable; it should be kept in mind that at the beginning we said that the charge $q$ would be considered negative, i.e., $q=-z e$, and as such it is used, that's why $\sqrt{-1}=\mathbf{i}$, the factors $z$ and $Z$ theoretically do not necessarily have to be an integer:

$$
s_{0}=|\sqrt{\sigma(\chi)(z Z)}|=\text { Const. } 3=\text { constant. }
$$

This size $S_{0}$ is a constant [12]; the higher is $z$ or $Z$, the smaller is $\sigma(\chi)$ [12], and vice versa, their product $\sigma(\chi)(z Z)$ is always the same [12]. These factors $z$ and $Z$ basically determine the system being observed. We say basically because the actual system is determined even with orbit parameters ( $n$; which we will see later) that are not expressed in Eq. (34), but those parameters are hidden in the members tagged with $E_{e m}$. It should be said, for the realization of the oscillator must be some charge present, but the amount of natural frequencies of these oscillators must not depend on the amount of these charges as a variable. So, from Eq. (33) and (34) we get:

$$
f=\frac{E_{e m}}{\sqrt{\mu_{0} / \varepsilon_{0}} e^{2} s_{0}^{2}} \frac{1-E_{e m} / 2 m c^{2}}{1-E_{e m} / m c^{2}} .
$$

It's in vacuum $1 / \sqrt{\mu_{0} \varepsilon_{0}}=c$ and $\sqrt{\mu_{0} / \varepsilon_{0}}=\mu_{0} c$, so from Eqs. $(23,30,34)$ follows:

$$
\begin{aligned}
& A=-\pi \sqrt{\frac{L}{C}} q Q \frac{1-E_{e m} / m c^{2}}{1-E_{e m} / 2 m c^{2}} \\
& =-\pi \sqrt{\frac{\mu_{0}}{\varepsilon_{0}}} \frac{\sigma(\chi)}{\pi} q Q \frac{1-E_{e m} / m c^{2}}{1-E_{e m} / 2 m c^{2}} \\
& =\sqrt{\frac{\mu_{0}}{\varepsilon_{0}}}[\sigma(\chi) z Z] e^{2} \frac{1-E_{e m} / m c^{2}}{1-E_{e m} / 2 m c^{2}} \\
& =\mu_{0} c e^{2} s_{0}^{2} \frac{1-E_{e m} / m c^{2}}{1-E_{e m} / 2 m c^{2}}=A_{0} \frac{1-E_{e m} / m c^{2}}{1-E_{e m} / 2 m c^{2}} .
\end{aligned}
$$


Here is

$$
A_{0}=\sqrt{\frac{\mu_{0}}{\varepsilon_{0}}} e^{2} s_{0}^{2}=\mu_{0} c e^{2} s_{0}^{2}
$$

the maximum of action of $L C$ oscillator, i.e., the action by $f=0$ respectively by $E_{e m}=0$. From Eqs. $(35,37)$, we get

$$
E_{e m}=A_{0} f \frac{1-E_{e m} / m c^{2}}{1-E_{e m} / 2 m c^{2}},
$$

and from Eq. (38) comes

$$
E_{e m}=A_{0} f+m c^{2}-\sqrt{\left(A_{0} f\right)^{2}+\left(m c^{2}\right)^{2}}
$$

and since Eq. (22) $E_{e m}=A f$, then

$$
A=A_{0}+m c^{2} / f-\sqrt{A_{0}^{2}+\left(m c^{2} / f\right)^{2}}
$$

Duane-Hunt's law in extended form [13] comes from Eq. (38), using Eq. (8), i.e., $E_{e m}=-q V_{e m}$ :

$$
f=-\frac{q V_{e m}}{A_{0}} \frac{1+q V_{e m} / 2 m c^{2}}{1+q V_{e m} / m c^{2}} .
$$

Namely, taking into account Eqs. $(8,36)$, Eq. (41) can be written as

$$
f=-\frac{q V_{e m}}{A}
$$

while the original form of Duane-Hunt's Law [14] is $f=e V_{e m} / h$; here $h$ is Planck's constant, which is replaced here by A, i.e., $h=A$.

Do we include $q V_{e m}=-m c^{2}\left(1-\sqrt{1-\beta^{2}}\right)$, from Eq. (8), in the Eq. (41), we get [using Eq. (37)]:

$$
f=\frac{m c^{2}}{2 A_{0}} \frac{\beta^{2}}{\sqrt{1-\beta^{2}}}=\frac{f_{0}}{2} \frac{\beta^{2}}{\sqrt{1-\beta^{2}}},
$$

here, respectively, $f_{0}$ and $\tau_{0}$ are the elementary frequency and the elementary time of one electron in an atom:

$$
\begin{aligned}
& f_{0}=\frac{m c^{2}}{A_{0}}=\frac{m c}{\mu_{0} e^{2} s_{0}^{2}}=1.23525211244 \times 10^{20} \mathrm{~Hz}, \\
& \tau_{0}=\frac{\beta^{2}}{2 f \sqrt{1-\beta^{2}}}=\frac{A_{0}}{m c^{2}}=\frac{1}{f_{0}}=\frac{\mu_{0} e^{2} s_{0}^{2}}{m c}=8.09551337682 \times 10^{-21} \mathrm{~s} .
\end{aligned}
$$

The Eq. (43), according to the Eqs. $(6,44)$, gives:

$$
f=-\frac{1}{2} \frac{U}{A_{0}}=-\frac{1}{2} \frac{U}{\mu_{0} c e^{2} s_{0}^{2}}
$$

and this leads to the Ritz's combination principle [15], $f_{k i}=\left[E_{e m(k)}-E_{e m(i)}\right] / h$, which in our case reads

$$
f_{k i}=\left(-\frac{1}{2} U_{k}+\frac{1}{2} U_{i}\right) / A_{0}
$$

where $U_{k}>U_{i}$. From Eq. (43) $\beta$ also can be written as 


$$
\beta=\sqrt{2} \sqrt{\sqrt{\left(\frac{f}{f_{0}}\right)^{4}+\left(\frac{f}{f_{0}}\right)^{2}}-\left(\frac{f}{f_{0}}\right)^{2}} .
$$

Now we have enough elements to go back and complete Eq. (27). Namely, from Eqs. (43, 44), the unknown relationship in Eq. (27) $m c^{2} \beta^{2} /\left(2 f \sqrt{1-\beta^{2}}\right)$, now reads as $A_{0}$, i.e.,

$$
\frac{m c^{2} \beta^{2}}{2 f \sqrt{1-\beta^{2}}}=A_{0} ; \quad \frac{\beta^{2}}{f \sqrt{1-\beta^{2}}}=\frac{2 A_{0}}{m c^{2}}=2 \tau_{0}=T_{0}=1.61910267536 \times 10^{-20} \mathrm{~s} .
$$

So, include it in Eq. (27), we get:

$$
A=A_{0} \frac{1-E_{e m} / m c^{2}}{1-E_{e m} / 2 m c^{2}}=\mu_{0} c e^{2} s_{0}^{2} \frac{1-E_{e m} / m c^{2}}{1-E_{e m} / 2 m c^{2}} .
$$

We see that we get the same result as the one at the end of the Eq. (36), what has to be so, and by the bypass we get in Eq. (47), i.e., the relation between $\beta$ and $f$, which is not entirely simple.

\section{Discretization of the States in the Atom}

Structural constant of the atom $s_{0}$ can be calculated in several ways $[12,16,17]$. Now we will see a new method. The momentum of the electromagnetic wave is defined as the photon momentum [15]

$$
p_{e m}=\frac{E_{e m}}{u_{e m}}
$$

whereby

$$
u_{e m}=c=\lambda f
$$

is phase velocity, and $\lambda$ is wavelength of electromagnetic wave. Using Eq. (42), i.e., $-q V_{e m}=A f$, and taking into account Eq. (8), $E_{e m}=-q V_{e m}$, and Eq. (51), $u_{e m}=\lambda f$, Eq. (50) takes the form of de Broglie's expression for the photon momentum [15]:

$$
p_{e m}=\frac{A f}{\lambda f}=\frac{A}{\lambda} .
$$

The photon momentum, in accordance with the law of conservation of momentum [8], and with Eq. (2), is equal in amount to the momentum of the electron. So Eq. (52) becomes:

$$
\frac{A}{\lambda}=m v / \sqrt{1-\beta^{2}}=m c \beta / \sqrt{1-\beta^{2}} .
$$

At least two independent physical phenomena appear in the atom, which after the transition process enable atomic formation. One is the uniform circular motion of the electron around the nucleus, and the second are the oscillations of the electromagnetic energy generated within the atom. The time of one complete revolution of the electron around the nucleus (the so-called period) is

$$
T_{\phi}=\frac{2 r \pi}{v}=\frac{1}{\phi}
$$

$\phi$ is the frequency of the rotation of body charged with the charge $q$. Entirely different oscillation period, period of electromagnetic oscillation, $T_{e m}$, is

$$
T_{e m}=\frac{1}{f} .
$$

The multiplication of the Eq. (54), with the frequency $f$ of electromagnetic oscillations, gives 


$$
\frac{2 r \pi f}{v}=\frac{f}{\phi} .
$$

Using Eq. (45), $f=-U /\left(2 A_{0}\right)$, and Eq. (6), $U=q Q /\left(4 \pi \varepsilon_{0} r\right)$, and taking into account that $\varepsilon_{0}=1 /\left(\mu_{0} c^{2}\right)$, and Eq. (37), $A_{0}=\mu_{0} c e^{2} s_{0}^{2}$, from Eq. (56) follows:

$$
\frac{f}{\phi}=-\frac{q Q}{4 \varepsilon_{0} A_{0} v}=-\frac{q Q c}{4 v e^{2} s_{0}^{2}} .
$$

Electromagnetic energy in the atom can exist as a standing wave. The standing wave does not transmit the energy, but it sways existing energy. If the natural frequency of the $L C$ oscillator is $f$, the active power (in the index marked with "AcP") of the standing wave oscillates with dual frequency [18], $f_{A c P}=2 f$. Thesis: In order for the electromagnetic standing wave to exist in the atom, there must be a mutual synchronization relationship between the frequency of electron motion around the nucleus and the frequency of oscillation of electromagnetic energy in the atom (it should be noted here that other integer relations between these two phenomena are theoretically possible):

$$
f_{A c P}=n \phi,
$$

where $n$ is one of the whole numbers $1,2,3, \ldots$. Both above mentioned phenomena in respect of synchronization are equal, so also applies

$$
\phi=n f_{A c P} .
$$

The two Eqs. $(58,59)$, can be written as one expression:

$$
f_{A c P}=n^{ \pm 1} \phi,
$$

or, because of $f_{A c P}=2 f$,

$$
f=\frac{1}{2} n^{ \pm 1} \phi .
$$

From Eqs. $(57,61)$ we obtain the velocity for discrete states:

$$
v_{n}=-\frac{c}{2 n^{ \pm 1} s_{0}^{2}} \frac{q Q}{e^{2}}=-\frac{c}{2 n^{ \pm 1} s_{0}^{2}} \frac{(-z e)(+Z e)}{e^{2}}=\frac{c z Z}{2 n^{ \pm 1} s_{0}^{2}} .
$$

From Eq. (61), after connecting $f=f_{n}$ and $\phi=\phi_{n}$, follows for frequencies in discrete states:

$$
f_{n}=\frac{1}{2} n^{ \pm 1} \phi_{n} .
$$

From Eq. (62), taking into account $v_{n}=c \beta_{n}$, we obtain:

$$
\beta_{n}=-\frac{1}{n^{ \pm 1}} \frac{q Q}{2 s_{0}^{2} e^{2}}=\frac{z Z}{2 n^{ \pm 1} s_{0}^{2}} .
$$

We can express $\beta$ from Eq. (8), $\beta=\sqrt{-q V_{e m}\left(2+q V_{e m} / m c^{2}\right) / m c^{2}}$, and equate this $\beta$ with that in the expression (64):

$$
\sqrt{-\frac{q V_{e m}}{m c^{2}}\left(2+\frac{q V_{e m}}{m c^{2}}\right)}=-\frac{1}{n^{ \pm 1}} \frac{q Q}{2 s_{0}^{2} e^{2}} .
$$

From Eq. (65), we get structural constant $s_{0}$ determined by the aforementioned new method, which proves to be the most accurate: 


$$
s_{0}=-\frac{\mathbf{i} \sqrt{\frac{q Q}{e^{2}}}}{\sqrt{2 n^{ \pm 1} \sqrt{1-\left(1+\frac{q V_{e m(n)}}{m c^{2}}\right)^{2}}}}
$$

Two $V_{e m(n)}$ solutions we get from Eq. (66):

$$
V_{e m 1(n)}=\frac{m c^{2}}{z e}\left(1-\sqrt{1-\left(\frac{1}{n^{ \pm 1}} \frac{z Z}{2 s_{0}^{2}}\right)^{2}}\right)
$$

and

$$
V_{e m 2(n)}=\frac{m c^{2}}{z e}\left(1+\sqrt{1-\left(\frac{1}{n^{ \pm 1}} \frac{z Z}{2 s_{0}^{2}}\right)^{2}}\right) .
$$

The solution in Eq. (67) is for the lower (ionization) voltages (namely, for $q=-e$, than to $510998.946 \mathrm{~V}$ ), and the solution in Eq. (68) is for the higher (ionization) voltages (so, for $q=-e$, than to $1021997.813 \mathrm{~V}$ ). We can see that $V_{e m 1(n)}+V_{e m 2(n)}=-2 m c^{2} / q$. Let us introduce now one abbreviation. Namely, instead of $\sqrt{1-\beta^{2}}$, in accordance with Eq. (64), we put $\Gamma_{n}$ (symbol of the field of Discrete Physics):

$$
\Gamma_{n}=\sqrt{1-\left(\frac{1}{n^{ \pm 1}} \frac{z Z}{2 s_{0}^{2}}\right)^{2}} .
$$

By incorporating Eqs. $(64,69)$ in Eq. (4), using $q=-z e, Q=Z e, \varepsilon_{0}=1 /\left(\mu_{0} c^{2}\right)$, it yields a radius of electron orbit:

$$
r_{n}=\left(n^{ \pm 1}\right)^{2} \frac{\mu_{0} e^{2} s_{0}^{4}}{\pi m z Z} \Gamma_{n} .
$$

So for example, repeat the calculation as we did after Eq. (13), but now in a different way; we calculate the radius of the first Hydrogen orbit. There we should measure $V_{e m}$ and used 6 more parameters; i.e., $z, Z, e, \mu_{0}, m, c$; while now, instead of measuring $V_{e m}$, we have the computational value $s_{0}$ and we need, also, 6 additional parameters; 5 of these parameters are the same as before, i.e., $z, Z, e, \mu_{0}, m$, and instead of the parameter $c$, we now use the number $n$. We get the same result without measuring $V_{e m}$ and we do not have to use the speed of light. Let's show this using equation (70). For the first orbit of Hydrogen $(z=1, Z=1, n=1), r_{\mathrm{H}}=5.294526279 \times 10^{-11} \mathrm{~m}$, and we get the radius of the first orbit of Helium $(z=1, Z=2, n=1), r_{\mathrm{He}}=2.647051780 \times 10^{-11} \mathrm{~m}$. Also we get the first orbit of Neon $(z=1, Z=10$, $n=1), \quad r_{\mathrm{Ne}}=5.280558679 \times 10^{-12} \mathrm{~m}$, while for Darmstadtium the first orbit is $(z=1, Z=110, n=1), \quad r_{\mathrm{Ds}}=$ $2.871955104 \times 10^{-13} \mathrm{~m}$. So, by introducing $s_{0}$ we avoided the measurements of $V_{e m}$ and got all the same results as before. The resulting deviations are most commonly inside $\pm 0.1 \%$ and at Darmstadtium $(Z=110)$ reach a maximum of $1.17 \%$. This difference probably stems from the accuracy of the measurements at such a large $V_{e m}$.

From Eq. (6) it has been generated the Coulomb potential, $V_{U}=Q /\left(4 \pi \varepsilon_{0} r\right)$, and from here, using Eq. (69), we get (with $q=-z e, Q=Z e$ ):

$$
V_{U(n)}=\frac{m c^{2} z Z^{2}}{4\left(n^{ \pm 1}\right)^{2} e s_{0}^{4} \Gamma_{n}} .
$$

Let's now calculate the impedances ratio $\gamma$. We start from Eq. (24): 


$$
\mathrm{Z}_{L C}=\sqrt{\frac{L}{C}}=\gamma Z_{0}=\gamma \mu_{0} c=\frac{1}{2 \pi f C}
$$

From here we get $\gamma$ :

$$
\gamma=\frac{\sqrt{L / C}}{\mu_{0} c}=\frac{1}{2 \pi f C \mu_{0} c} .
$$

Now we have to determine $C$ and $f$. So we use Eq. (20), $C=4 \pi \varepsilon_{0} r$, and Eq. (45), $f=-U /\left(2 \mu_{0} c e^{2} s_{0}^{2}\right)$, than from Eq. (73) we get:

$$
\gamma=-\frac{e^{2} s_{0}^{2}}{4 \pi^{2} \varepsilon_{0} r U} .
$$

Using Eq. (6); $U=q Q /\left(4 \pi \varepsilon_{0} r\right)$, we get from Eq. (74):

$$
\gamma=-\frac{e^{2} s_{0}^{2}}{\pi q Q} .
$$

If we use $q=-z e$ and $Q=+Z e$, from Eq. (75) we get:

$$
\gamma=\frac{s_{0}^{2}}{\pi z Z}
$$

From Eq. (72), $\gamma=\sqrt{L / C} /\left(\mu_{0} c\right)$, and Eq. (75), $\gamma=s_{0}^{2} /(\pi z Z)$, derives

$$
s_{0}=\sqrt{\frac{z Z \pi \sqrt{L / C}}{\mu_{0} c}} .
$$

In Eq. equation (77) one of its parts, i.e., $z Z \pi \sqrt{L / C}$, is equal $\mu_{0} c s_{0}^{2}$, which corresponds in amount to von Klitzing's constant $R_{\mathrm{K}}$. Deeper connection with the von Klitzing constant needs to be investigated.

If we equate Eq. (41), for the frequencies, $f=-\left[q Q\left(1+q V_{e m} / 2 m c^{2}\right)\right] /\left[A_{0}\left(1+q V_{e m} / m c^{2}\right)\right]$, and Eq. (45); $f=-U /\left(2 \mu_{0} c e^{2} s_{0}^{2}\right)$, and then use Eq. (6); $U=-m c^{2} \beta^{2} / \sqrt{1-\beta^{2}}$ and Eq. (36); $A_{0}=\mu_{0} c e^{2} s_{0}^{2}$, we can calculate the (ionization) voltage $V_{e m}$ depending on $\beta$ :

$$
V_{e m}=-\frac{m c^{2}}{q} \frac{\beta^{2} m c^{2}+2 \sqrt{1-\beta^{2}} q Q}{\beta^{2} m c^{2}+\sqrt{1-\beta^{2}} q Q} .
$$

If $\beta=1$ the (ionization) voltage is maximum, and is: $V_{e m}=V_{\max }=-m c^{2} / q$.

Now from Eq. (66) we can calculate minimum of $n^{ \pm 1}, n_{\min }^{ \pm 1}$, and maximum of $Z, Z_{\max }$. So, if we take $z=1$ and $Z=1$, it comes out $n_{\min }^{ \pm 1}=-q Q /\left(2 e^{2} s_{0}^{2}\right)=-(-z e)(+Z e) /\left(2 e^{2} s_{0}^{2}\right)=1 /\left(2 s_{0}^{2}\right)$.

Similarly, if we take $z=1$ and $n^{ \pm 1}=1$, we get from Eq. (66) the highest atomic number $Z_{\text {max }}=-2 e n^{ \pm 1} s_{0}^{2} / q=-2 e s_{0}^{2} /(-e)=2 s_{0}^{2}$.

If we introduce Eqs. $(64,69)$ into Eq. $(6)$, we get the potential energy of the system:

$$
U_{n}=-\frac{m c^{2} q^{2} Q^{2}}{4\left(n^{ \pm 1}\right)^{2} e^{4} s_{0}^{4} \sqrt{1-\beta_{n}^{2}}}=-\frac{m c^{2} z^{2} Z^{2}}{4\left(n^{ \pm 1}\right)^{2} s_{0}^{4} \Gamma_{n}} .
$$

The kinetic energy of the system is derived from Eqs. $(5,64)$ : 


$$
K_{n}=m c^{2}\left(\frac{1}{\sqrt{1-\beta_{n}^{2}}}-1\right)=m c^{2}\left(\frac{1}{\Gamma_{n}}-1\right) .
$$

The mechanical energy of the system $W$, according to equation (7), then amounts:

$$
W_{n}=m c^{2}\left(\sqrt{1-\beta_{n}^{2}}-1\right)=m c^{2}\left(\Gamma_{n}-1\right) .
$$

From there, the energy of the system, $E_{e m(n)}=-W_{n}=-q V_{e m(n)}$, according to the equation (8), is:

$$
E_{\text {em }(n)}=-q V_{e m(n)}=-m c^{2}\left(\sqrt{1-\beta_{n}^{2}}-1\right)=m c^{2}\left(1-\Gamma_{n}\right) .
$$

The electromagnetic frequency is obtained from Eqs. $(45,79)$ :

$$
f_{n}=\frac{m c q^{2} Q^{2}}{8\left(n^{ \pm 1}\right)^{2} \mu_{0} e^{6} s_{0}^{6} \sqrt{1-\beta_{n}^{2}}}=\frac{m c z^{2} Z^{2}}{8\left(n^{ \pm 1}\right)^{2} \mu_{0} e^{2} s_{0}^{6} \Gamma_{n}} .
$$

From Eqs. $(27,64,81,83)$, we obtain the action $A_{n}$ of the electromagnetic oscillator:

$$
A_{n}=\mu_{0} c e^{2} s_{0}^{2} \frac{2 \sqrt{1-\beta_{n}^{2}}}{1+\sqrt{1-\beta_{n}^{2}}}=\mu_{0} c e^{2} s_{0}^{2} \frac{2 \Gamma_{n}}{1+\Gamma_{n}} .
$$

The capacitance $C$ is derived from Eqs. (20, 70):

$$
C_{n}=-\frac{\left(2 n^{ \pm 1} e^{2} s_{0}^{2}\right)^{2}}{m c^{2} q Q} \sqrt{1-\beta_{n}^{2}}=\frac{\left(2 n^{ \pm 1} e s_{0}^{2}\right)^{2}}{m c^{2} z Z} \Gamma_{n} .
$$

The inductance $L$ can be expressed from equation (16) with the help of Eqs. (83, 85):

$$
L_{n}=-\frac{\left(2 n^{ \pm 1} \mu_{0} e^{4} s_{0}^{4}\right)^{2}}{m \pi^{2} q^{3} Q^{3}} \sqrt{1-\beta_{n}^{2}}=\frac{\left(2 n^{ \pm 1} \mu_{0} e s_{0}^{4}\right)^{2}}{m \pi^{2} z^{3} Z^{3}} \Gamma_{n} .
$$

For the first orbit of a hydrogen atom $(n=z=Z=1)$ it is worth: $C_{1}=5.890954960 \times 10^{-21} \mathrm{~F}, L_{1}=3.979183560 \times 10^{-13} \mathrm{H}$, $\sqrt{L_{1} / C_{1}}=8218.719068068 \Omega, s_{0}=\sqrt{z Z \pi \sqrt{L_{1} / C_{1}} /\left(\mu_{0} c\right)}=8.278691910$.

In order to verify the previous equations, add Eqs. $(85,86)$ to Eq. $(77)$. We get structural constant $s_{0}$. So, everything is correct.

\section{The Numerical Value of the Structural Constant}

Derived formulas do not allow direct calculation of structural constant $s_{0}$. So we have to do at least one measurement. This can be best done through Eq. (66), thanks to the precision of NIST data [9],

[https://www.nist.gov/pml/atomic-spectra-database; $V_{e m(1)}=13.59843449 \mathrm{~V}, n^{ \pm 1}=1, \quad z=1, Z=1$ ]:

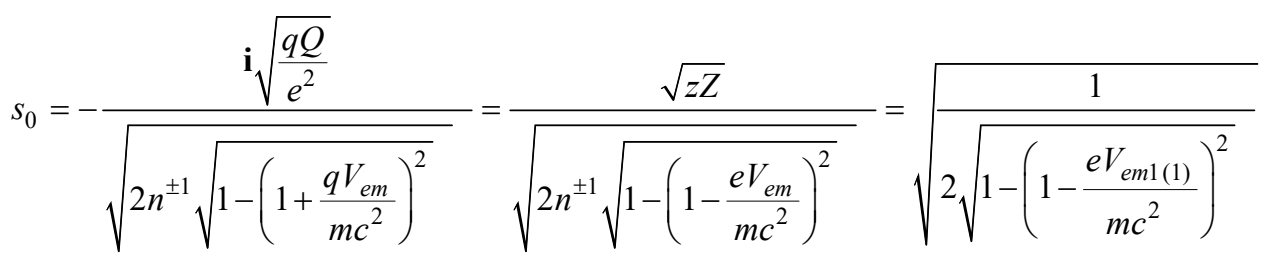

$=8.278691910036$. 
Table 1. Structural constant of atoms $s_{0}$ calculated on the basis of ionization potential according to NIST's data*

\begin{tabular}{|c|c|c|c|c|c|}
\hline \multirow{2}{*}{$\begin{array}{l}\text { Chemical } \\
\text { symbol of the } \\
\text { element }\end{array}$} & \multicolumn{2}{|c|}{ Atomic number } & \multirow{2}{*}{$\begin{array}{c}\text { Ionization potential } \\
{[\text { Volt }]}\end{array}$} & & \multirow{2}{*}{$\begin{array}{c}\text { Structural constant } s_{0} \\
\text { [Dimensionless Number], } \\
\text { Eq. (87) }\end{array}$} \\
\hline & $Z$ & Eq. (68), neutron ${ }^{a}$ & & & \\
\hline $\mathrm{n}^{0}, \mathrm{H}$ & 1 & $712207.8053 \mathrm{~V}$ & $13.59843449^{b}$ & $\leftrightarrow$ & 8.278691910036 \\
\hline $\mathrm{He}$ & 2 & & $54.4177650^{\mathrm{b}}$ & $\leftarrow$ & 8.277860595602 \\
\hline $\mathrm{Li}$ & 3 & & $122.4543581^{b}$ & $\leftrightarrow$ & 8.277755226469 \\
\hline $\mathrm{Be}$ & 4 & & $217.7185843^{b}$ & $\leftrightarrow$ & 8.277739533105 \\
\hline B & 5 & & $340.226020^{\mathrm{b}}$ & $\leftrightarrow$ & 8.277739896484 \\
\hline $\mathrm{C}$ & 6 & & $489.993194^{b}$ & $\leftrightarrow$ & 8.277757231963 \\
\hline $\mathrm{N}$ & 7 & & $667.046116^{\mathrm{b}}$ & $\leftrightarrow$ & 8.277771755296 \\
\hline $\mathrm{O}$ & 8 & & $871.40988^{b}$ & $\leftrightarrow$ & 8.277791688375 \\
\hline $\mathrm{F}$ & 9 & & $1103.11747^{b}$ & $\leftrightarrow$ & 8.277812276144 \\
\hline $\mathrm{Ne}$ & 10 & & $1362.19915^{b}$ & $\leftrightarrow$ & 8.277842618038 \\
\hline $\mathrm{Ca}$ & 20 & & $5469.8615^{b}$ & $\leftrightarrow$ & 8.278203152859 \\
\hline $\mathrm{Zn}$ & 30 & & $12388.929^{b}$ & $\leftrightarrow$ & 8.278637976575 \\
\hline $\mathrm{Zr}$ & 40 & & $22236.677^{b}$ & $\leftrightarrow$ & 8.279106265650 \\
\hline $\mathrm{Sn}$ & 50 & & $35192.39^{b}$ & $\leftrightarrow$ & 8.279610860584 \\
\hline $\mathrm{Nd}$ & 60 & & $51515.58^{b}$ & $\leftarrow \rightarrow$ & 8.280166600276 \\
\hline $\mathrm{Yb}$ & 70 & & $71574.80^{\mathrm{b}}$ & $\leftrightarrow$ & 8.280846679237 \\
\hline $\mathrm{Hg}$ & 80 & & $95897.70^{b}$ & $\leftrightarrow$ & 8.281775555833 \\
\hline Th & 90 & & $125253.40^{\mathrm{b}}$ & $\leftrightarrow$ & 8.283267729872 \\
\hline $\mathrm{Fm}$ & 100 & & $160804.00^{b}$ & $\leftrightarrow$ & 8.286011987216 \\
\hline Ds & 110 & & $204400.00^{b}$ & $\leftrightarrow$ & 8.291558770012 \\
\hline $\mathrm{Rg}$ & 111 & & $211181.96^{c}$ & $\leftarrow$ & $8.278691910036^{d}$ \\
\hline $\mathrm{Cn}$ & 112 & & $216395.64^{\mathrm{c}}$ & $\leftarrow$ & $8.278691910036^{d}$ \\
\hline $\mathrm{Fl}$ & 114 & & $227257.00^{\mathrm{c}}$ & $\leftarrow$ & $8.278691910036^{d}$ \\
\hline $\mathrm{Lv}$ & 116 & & $238755.14^{\mathrm{c}}$ & $\leftarrow$ & $8.278691910036^{d}$ \\
\hline $\mathrm{Og}$ & 118 & & $250947.50^{\mathrm{c}}$ & $\leftarrow$ & $8.278691910036^{d}$ \\
\hline$x x^{e}$ & 119 & & $257386.97^{\mathrm{c}}$ & $\leftarrow$ & $8.278691910036^{d}$ \\
\hline$x x^{e}$ & 120 & & $264022.11^{c}$ & $\leftarrow$ & $8.278691910036^{d}$ \\
\hline $\mathrm{xx}^{\mathrm{e}}$ & 122 & & $278037.10^{\mathrm{c}}$ & $\leftarrow$ & $8.278691910036^{d}$ \\
\hline$x x^{e}$ & 126 & & $309790.06^{c}$ & $\leftarrow$ & $8.278691910036^{d}$ \\
\hline$x x^{e}$ & 130 & & $348967.80^{\mathrm{c}}$ & $\leftarrow$ & $8.278691910036^{d}$ \\
\hline$x x^{e}$ & 132 & & $373260.57^{c}$ & $\leftarrow$ & $8.278691910036^{d}$ \\
\hline$x x^{e}$ & 134 & & $403395.73^{c}$ & $\leftarrow$ & $8.278691910036^{d}$ \\
\hline$x x^{e}$ & 136 & & $447172.13^{c}$ & $\leftarrow$ & $8.278691910036^{d}$ \\
\hline $\mathrm{xx}^{\mathrm{e}}$ & 137 & & $494269.44^{c}$ & $\leftarrow$ & $8.278691910036^{d}$ \\
\hline
\end{tabular}

*NIST: National Institute of Standards and Technology.

${ }^{a}$ Neutron, according to the assumption herein, it is treated as hydrogen atom in the discrete state $n^{-1}=126$, according to Eq. (68), with $z=Z=1$. https://physics.nist.gov/PhysRefData/ASD/ionEnergy.html

${ }^{\mathrm{b}}$ The data is from NIST Atomic Spectra Database Ionization Energies Form;

'No data are available by NIST (June 30, 2020); the amount of $V_{\mathrm{em}}$ obtained by using Eq. (82), which can also be calculated from equation (87).

${ }^{\mathrm{d}}$ The structural constant of hydrogen is used here, Eq. (87); $s_{0}=8.278691910036$.

${ }^{\mathrm{e}}$ The element has not been revealed yet, but in accordance with here presented theory the highest atomic number can be $Z=137$. 


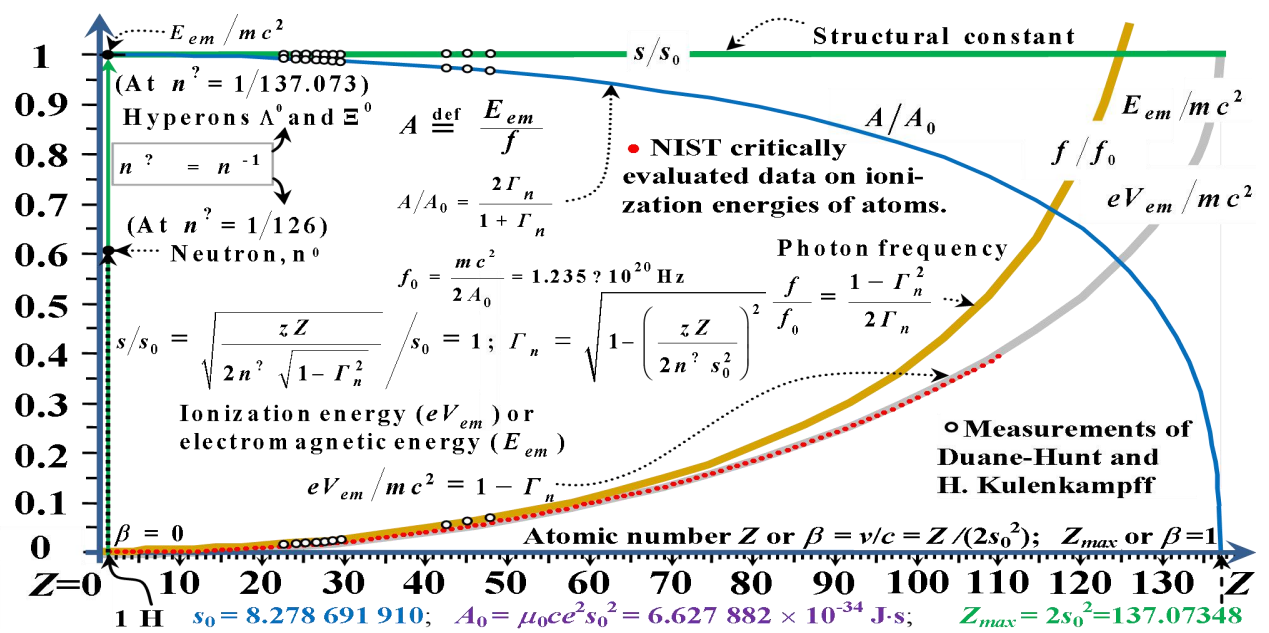

Figure 2. a) Electromagnetic energy of photon $E_{e m}$, ionization energy of electron $e V_{e m}$, photon frequency $f$, structural constant $s$, and $L C$ oscillator action $A$, versus atomic number $Z$ or electron speed $\beta=v / c ; n=1$; Measurements marked with "•" are from

NIST, the remaining measurements, marked with "o", are from Duane-Hunt [14] and H. Kulenkampff [19].

\section{Application of Structural Constant}

The use of the structural constant has many applications. Here we will highlight three such applications in particular. One application is that the structural constant makes 9 existing constants redundant (Table 2). Another application of the structural constant is the transfer of continuous theory to discrete theory or Discrete Physics (Table 3). The third application of the structural constant, on which the introduction of the unit for the type of substance is based, 'boscovich', $\mathrm{B}=1 /\left(2 s_{0}^{2}\right),[20]$.

The value of structural constant of atoms $s_{0}$ is the same for all atoms (Table 1). This is a Fundamental Physical Constant, (perhaps even in the category "Universal Constants", next to characteristic impedance of vacuum, electric constant, magnetic constant, Newtonian constant of gravitation, Planck constant, or speed of light in vacuum). When we know $s_{0}$ then we can from Eqs. $(67,68)$ calculate $V_{e m 1(n)}, V_{e m 2(n)}$, and all other sizes (Figure 2).

Structural constant can also be calculated in the following ways. The simplest way is to use von Klitzing constant, i.e., $R_{\mathrm{K}}=25812.8074555 \Omega$, CODATA2014 https://physics.nist.gov/cgi-bin/cuu/Value?rk, stimulated by the Eq. (77):

$$
s_{0}=\sqrt{\frac{R_{\mathrm{K}}}{\mu_{0} c}}=8.277560000946 .
$$

Furthermore, for determining $s_{0}$ it is possible to use Planck's constant $\left(h=6.626070040 \times 10^{-34} \mathrm{~J} \cdot \mathrm{s}\right)$ :

$$
s_{0}=\sqrt{\frac{h}{\mu_{0} c e^{2}}}=8.277560001045 .
$$

It is also possible to use Josephson's constant $\left(K_{\mathrm{J}}=483597.8525 \times 10^{9} \mathrm{~Hz} \cdot \mathrm{V}^{-1}\right)$ :

$$
s_{0}=\sqrt{\frac{2}{\mu_{0} c e K_{\mathrm{J}}}}=8.277560000946 .
$$

Finally, that $S_{0}$ can also be calculated using Rydberg's constant $\left(R_{\infty}=10973731.568508 \mathrm{~m}^{-1}\right)$

$$
s_{0}=\sqrt[6]{\frac{m}{8 \mu_{0} e^{2} R_{\infty}}}=8.277560001301 .
$$


Table 2. Six initial constants $\left(s_{0}, c, \mu_{0}, e, m, m_{\mathrm{p}}\right)$ convert nine below displayed constants in redundant

\begin{tabular}{|c|c|c|c|c|c|}
\hline Quantity & Symbol & Formula & Value & Unit & Difference $^{a}$ \\
\hline Structural constant & $s_{0}$ & $s_{0}$ & $8.278691910036^{\mathrm{b}}$ & 1 & unknown \\
\hline Speed of light in vacuum & $c$ & $c$ & 299792458 & $\mathrm{~m} \cdot \mathrm{s}^{-1}$ & 0.000 \\
\hline Magnetic constant & $\mu_{0}$ & $\mu_{0}$ & $1.256637061 \times 10^{-7}$ & $\mathrm{~kg} \cdot \mathrm{m} \cdot \mathrm{A}^{-2} \cdot \mathrm{s}^{-2}$ & 0.000 \\
\hline Elementary charge & $e$ & $e$ & $1.602176621 \times 10^{-19}$ & $A \cdot s$ & 0.000 \\
\hline Electron mass & $m$ & $m$ & $9.109383560 \times 10^{-31}$ & $\mathrm{~kg}$ & 0.000 \\
\hline \multirow[t]{2}{*}{ Proton mass } & $m_{\mathrm{p}}$ & $m_{\mathrm{p}}$ & $1.672621898 \times 10^{-27}$ & $\mathrm{~kg}$ & 0.000 \\
\hline & Down: & 9 redundant constants & derived from six initial & & \\
\hline 1. Fine-structure constant & $\alpha$ & $1 /\left(2 s_{0}^{2}\right)$ & $7.295357233 \times 10^{-3}$ & 1 & $-0.027^{c}$ \\
\hline 1a). Inverse fine-structure c. & $\alpha^{1}$ & $2 s_{0}^{2}$ & $1.370734795 \times 10^{2}$ & 1 & $+0.027^{\mathrm{c}}$ \\
\hline 2. von Klitzing constant & $R_{\mathrm{K}}$ & $\mu_{0} c s_{0}^{2}$ & $2.581986745 \times 10^{4}$ & $\mathrm{~m}^{2} \cdot \mathrm{kg} \cdot \mathrm{s}^{-3} \cdot \mathrm{A}^{-2}$ & $+0.027^{\mathrm{c}}$ \\
\hline 3. Planck's constant & $h$ & $\mu_{0} c e^{2} s_{0}^{2}$ & $6.627882313 \times 10^{-34}$ & $\mathrm{~m} \cdot \mathrm{kg} \cdot \mathrm{s}^{-1}$ & $+0.027^{\mathrm{c}}$ \\
\hline 3a). Conversion constant & $K_{0}$ & $1 /\left(2 \mu_{0} c e s s_{0}^{2}\right)$ & $1.208664053 \times 10^{14}$ & $\mathrm{~m}^{2} \cdot \mathrm{kg} \cdot \mathrm{s}^{-4} \cdot \mathrm{A}^{-1}$ & unknown \\
\hline 4. Ratio $e / h=2 K_{0}$ & $e / h$ & $1 /\left(\mu_{0} c\right.$ e $\left.s_{0}^{2}\right)$ & $2.417328106 \times 10^{14}$ & $\mathrm{~m}^{2} \cdot \mathrm{kg} \cdot \mathrm{s}^{-4} \cdot \mathrm{A}^{-1}$ & $-0.027^{c}$ \\
\hline 5. Josephson constant, $4 K_{0}$ & $K_{\mathrm{J}}$ & $2 /\left(\mu_{0} c\right.$ e $\left.s_{0}^{2}\right)$ & $4.834656212 \times 10^{14}$ & $\mathrm{~m}^{2} \cdot \mathrm{kg} \cdot \mathrm{s}^{-4} \cdot \mathrm{A}^{-1}$ & $-0.027^{c}$ \\
\hline 6. Rydberg constant & $R_{\infty}$ & $m /\left(8 \mu_{0} e^{2} s_{0}^{6}\right)$ & $1.096473231 \times 10^{7}$ & $\mathrm{~m}^{-1}$ & $-0.082^{c}$ \\
\hline 7. Bohr radius & $a_{0}$ & $\mu_{0} e^{2} s_{0}^{4} /(\pi m)$ & $5.294667174 \times 10^{-11}$ & $\mathrm{~m}$ & $+0.055^{\mathrm{c}}$ \\
\hline 8. Bohr magneton & $\mu_{\mathrm{B}}$ & $\mu_{0} c e^{3} s_{0}^{2} /(4 \pi m)$ & $9.276546489 \times 10^{-24}$ & $\mathrm{~A} \cdot \mathrm{m}^{2}$ & $+0.027^{\mathrm{c}}$ \\
\hline 9. Nuclear magneton & $\mu_{\mathrm{N}}$ & $\mu_{0} c e^{3} s_{0}^{2} /\left(4 \pi m_{\mathrm{p}}\right)$ & $5.052165117 \times 10^{-27}$ & $\mathrm{~A} \cdot \mathrm{m}^{2}$ & $+0.027^{\mathrm{c}}$ \\
\hline
\end{tabular}

aIt is the difference with "2014 CODATA recommended values" in percent. ${ }^{b}$ These calculation is based on the values provided by NIST, Eq. (87), $V_{e m 1(1)}=13.59843449 \mathrm{~V}$, https://www.nist.gov/pml/atomic-spectra-database. ${ }^{~}$ This difference disappears completely if Planck's constant instead of $h=6.626070040 \times 10^{-34} \mathrm{~J} \cdot \mathrm{s}$ is equal to $A_{0}=6.6278823131934 \times 10^{-34} \mathrm{~J} \cdot \mathrm{s}$, i.e., when it is increased by $0.027 \%$.

\section{Neutron as a hydrogen atom in discrete state $n^{-1}=126$}

If, for any reason, an electron falls below the first orbit $n^{ \pm 1}=1$, then the atom in that state can still exist. Suppose, therefore, that a neutron is in fact a hydrogen atom in one of the states $n^{-1}$. We will now determine which number $n$, below the level $n^{ \pm 1}=1$, belongs to that state of hydrogen atom or neutron. According to NIST, the mass of the proton is $m_{\mathrm{P}}=1.67262192369 \times 10^{-27} \mathrm{~kg}$, the mass of the neutron is $m_{\mathrm{n}^{0}}=1.67492749804 \times 10^{-27} \mathrm{~kg}$ and the mass of the electron is $m=9.1093837015 \times 10^{-31} \mathrm{~kg}$. The mass of hydrogen is, Eq. (69), $m_{\mathrm{H}}=m_{\mathrm{P}}+m / \sqrt{1-\beta_{\mathrm{H}}^{2}}=m_{\mathrm{P}}+m / \Gamma_{\mathrm{H}}=1.67353295896 \times 10^{-27} \mathrm{~kg}$. The difference between the mass of neutrons and the mass of hydrogen, $\quad \Delta m_{\mathrm{H}}=m_{\mathrm{n}^{0}}-m_{\mathrm{H}} \quad=1.39453907917 \times 10^{-30} \mathrm{~kg} \quad=\left(m_{\mathrm{P}}+m / \sqrt{1-\beta_{\mathrm{n}^{0}}^{2}}\right) \quad-\left(m_{\mathrm{P}}+m / \sqrt{1-\beta_{\mathrm{H}}^{2}}\right)$ $=m / \sqrt{1-\beta_{\mathrm{n}^{0}}^{2}}-m / \sqrt{1-\beta_{\mathrm{H}}^{2}}=m / \Gamma_{\mathrm{n}^{0}}-m / \Gamma_{\mathrm{H}}$, should then be attributed to the increase in the mass of electrons in the observed hydrogen atom as neutrons. It follows from here $\Gamma_{\mathrm{n}^{0}}=m \Gamma_{\mathrm{H}} /\left(\mathrm{m}+\Delta m_{\mathrm{H}} \Gamma_{\mathrm{H}}\right)$. According to Eq. (69), with $\mathrm{z}=\mathrm{Z}=n^{ \pm 1}=1$, is $\Gamma_{\mathrm{H}}=0.999973389$. Therefore, the solution of the previous equation, which is now, with the help of Eq. (69), written in extended form, [see Eq. (92)]. 
Table 3. Link of Continuous (Classical) and Discrete Physics

\begin{tabular}{|c|c|c|c|c|c|}
\hline Quantity & Symbol & Eq. & Continuously & Eq. & Discretely \\
\hline Structural constant & $s_{0}$ & (77) & $\sqrt{z Z \pi \sqrt{L / C} /\left(\mu_{0} c\right)}$ & (87) & $\sqrt{z Z /\left(2 n \pm 1 \sqrt{1-\Gamma_{n}^{2}}\right)}$ \\
\hline Particle velocity & $v$ & $(8) \div(9)$ & $\sqrt{-2 q V_{e m}\left[1+q V_{e m} /\left(2 m c^{2}\right)\right] / m}$ & (62) & $c z Z /\left(2 n^{ \pm 1} s_{0}^{2}\right)$ \\
\hline Ratio $v / c$ & $\beta$ & $(1) \div(2)$ & $v / c$ & (62) & $z Z /\left(2 n^{ \pm 1} s_{0}^{2}\right)$ \\
\hline Abbreviation & $\Gamma_{n}$ & (69) & $\sqrt{1-\beta^{2}}$ & (69) & $\sqrt{1-\left[z Z /\left(2 n^{ \pm 1} S_{0}^{2}\right)\right]^{2}}$ \\
\hline Ratio $(v / c)^{2}$ & $\beta^{2}$ & $(8) \div(9)$ & $(v / c)^{2}$ & (69) & $1-\Gamma_{n}^{2}$ \\
\hline Relativistic moment & $p$ & (2) & $m c \beta / \sqrt{1-\beta^{2}}$ & $(50)$ & $m c\left(1-\Gamma_{n}^{2}\right) / \Gamma_{n}$ \\
\hline Orbit radius & $r$ & (4) & $-q Q \sqrt{1-\beta^{2}} /\left(4 \pi \varepsilon_{0} \beta^{2} m c^{2}\right)$ & $(4,69)$ & $z Z \mu_{0} e^{2} \Gamma_{n} /\left[4 \pi m\left(1-\Gamma_{n}^{2}\right)\right]$ \\
\hline Orbit radius & $r$ & (13) & $\mu_{0} c^{2} Q\left[1+q V_{e m} /\left(m c^{2}\right)\right] /\left\{8 \pi V_{e m}\left[1+q V_{e m} /\left(2 m c^{2}\right)\right]\right\}$ & $(70)$ & $\left(n^{ \pm 1}\right)^{2} \mu_{0} e^{2} s_{0}^{4} \Gamma_{n} /(\pi m z Z)$ \\
\hline Kinetic energy & $K$ & (5) & $m c^{2} / \sqrt{1-\beta^{2}}-m c^{2}$ & (80) & $m c^{2}\left(1 / \Gamma_{n}-1\right)$ \\
\hline Potential energy & $U$ & (6) & $-m c^{2} \beta^{2} / \sqrt{1-\beta^{2}}$ & (79) & $m c^{2}\left(\Gamma_{n}-1 / \Gamma_{n}\right)$ \\
\hline Mechanical energy & $W$ & (7) & $-m c^{2}\left(1-\sqrt{1-\beta^{2}}\right)$ & (81) & $m c^{2}\left(\Gamma_{n}-1\right)$ \\
\hline Electromag. energy & $E_{e m}$ & (8) & $m c^{2}\left(1-\sqrt{1-\beta^{2}}\right)=-q V_{e m}$ & $(82)$ & $m c^{2}\left(1-\Gamma_{n}\right)$ \\
\hline Potential difference & $V_{e m}$ & $(67)$ & $-m c^{2}\left(1-\sqrt{1-\beta^{2}}\right) / q$ & $(67,68)$ & $m c^{2}\left(1 \pm \Gamma_{n}\right) /(z e)$ \\
\hline Coulomb pot., $U / q$ & $V_{U}$ & $(70 \div 71)$ & $Q /\left(4 \pi \varepsilon_{0} r\right)=U / q$ & (71) & $m c^{2}\left(1 / \Gamma_{n}-\Gamma_{n}\right) /(z e)$ \\
\hline Capacitance & $C$ & (20) & $4 \pi \varepsilon_{0} r$ & (85) & $\left(2 n^{ \pm 1}\right)^{2} e^{2} s_{0}^{4} \Gamma_{n} /\left(m c^{2} z Z\right)$ \\
\hline Inductance & $L$ & (16) & $1 /\left(4 \pi^{2} f^{2} C\right)$ & (86) & $z Z \mu_{0}^{2} e^{2} \Gamma_{n} /\left[\left(2 n^{ \pm 1} \pi\right)^{2} m\left(1-\Gamma_{n}^{2}\right)^{2}\right]$ \\
\hline Oscillator frequency & $f$ & $(42)$ & $1 /(2 \pi \sqrt{L C})=-q V_{e m} / A^{\mathrm{a}}$ & $(43,69)$ & $m c^{2}\left(1-\Gamma_{n}^{2}\right) /\left(2 A_{0} \Gamma_{n}\right)$ \\
\hline Action constant & $A_{0}$ & $(37,77)$ & $z Z \pi \sqrt{L / C} e^{2}$ & (37) & $\mu_{0} c e^{2} s_{0}^{2}$ \\
\hline$L C$ oscillator action & $A$ & $(8,42)$ & $E_{e m} / f=2 \pi m c^{2}\left(1-\sqrt{1-\beta^{2}}\right) \sqrt{L C}$ & $(40)$ & $A_{0}\left[2 \Gamma_{n} /\left(1+\Gamma_{n}\right)\right]$ \\
\hline Planck's $h$ is variable ${ }^{\mathrm{b}}$ & $h$ & $(36)$ & $E_{e m} / f$ & $(36,48)$ & $A_{0}\left[2 \Gamma_{n} /\left(1+\Gamma_{n}\right)\right]$ \\
\hline
\end{tabular}

at is simultaneously Duane-Hunt's law in expanded form.

${ }^{b}$ Definition of $h$ : The Planck constant, $h$, is equal to the energy $E_{e m}$ of a quantum of electromagnetic radiation divided by its frequency $f$, https://en.wikipedia.org/wiki/Planck_constant, see $A / A_{0} \Rightarrow h / A_{0}$ in Figure 2, from which it can be seen that $h$ is approximately constant up to the velocities of the body below $10 \%$ of the speed of light, and with increasing speed it decreases all the way to zero.

$$
\sqrt{1-\left(\frac{1}{n_{\mathrm{n} 0}^{ \pm 1}} \frac{z Z}{2 s_{0}^{2}}\right)^{2}}=\frac{\sqrt{1-\left(\frac{1}{n_{\mathrm{H}}^{ \pm 1}} \frac{z Z}{2 s_{0}^{2}}\right)^{2}}}{1+\frac{\Delta m_{\mathrm{H}}}{m} \sqrt{1-\left(\frac{1}{n_{\mathrm{H}}^{ \pm 1}} \frac{z Z}{2 s_{0}^{2}}\right)^{2}}}
$$

is $n_{\mathrm{n}^{0}}^{ \pm 1}=n_{\mathrm{n}^{0}}^{-1}=125.92000300221$. According to Eqs. $(59,60)$, here must be an integer, so that the real solution is $n_{\mathrm{n}^{0}}^{-1}=126$, where the error is $0.064 \%$. The ionization potential of neutrons is calculated from equation (68) and this ionization potential $\left(z=Z=1, n^{ \pm 1}=1 / 126\right)$ is $V_{e m_{n^{0}}}=712207.8053 \mathrm{~V}$. 


\section{Conclusion}

Maxwell's theory of electromagnetism and the relativity theory give here good results in describing most phenomena in the atom, such as the radiation of energy, discretization of the state in the atom, determination of stationary orbits, determining and calculating the structural constant of the atom. These theories enable for the transfer of continuous theory into the discrete theory of atoms. The discrete theory, apart from the description of the state in the electron shell, hence, in the higher orbits of the atoms, allows entry into the orbits that are theoretically present and under the first orbit of the atom, what lead towards the nuclei of atoms. The theory presented here explains that in atoms, in addition to discrete states $n^{+1}=1,2,3$, discrete states $n^{-1}=1,2,3$, are also present. Therefore, it is possible that in the discrete state $n^{-1}=126$ the hydrogen atom acquires the properties of a neutron. The resulting neutron then, with another proton, builds a nucleus of deuterium, tritium, and all others nucleus. Protons and electrons are then attracted to the nuclei, which overcome the mutual repulsive force of the protons. To achieve all this, the presence of an electromagnetic oscillator within the atom is assumed. This oscillator is described using the Lecher line. Discretization is introduced through the integer ratio of the natural frequency of electromagnetic oscillations in the atom and the frequency of rotation of the electron around the nucleus of the atom. This integer ratio allows stable states of the atom. The experimental results are in agreement with the presented theory. Introduced structural constant $s_{0}$ help to make redundant 9 other physical constants, it is in detail explained and this constant amounts 8.278691910. This structural constant allows other research to continue; one of them is the unit for type of substance. The exposition in this article is based on one-electron atoms. Multiple-electron atoms, on the foundations set up here, should be separate research. It is shown that Planck's $h$ is not constant, and that it can be considered constant only in cases when the speed of the observed body is lower than about $10 \%$ of the speed of light. At body speeds greater than $10 \%$ of the speed of light, Planck's $h$ gradually decreases even to zero. This article is written in a logical, physical, and formally clear sequence, so that all concepts are perfectly clear as much as they are clear in classical physics.

\section{Acknowledgements}

For fruitful discussions, I thank Tomislav Ivezić. For professional help and monitoring, thanks to Srebrenka Ursić, Damir Vuk, Jasna Gašparović, Branko Balon, Velibor Ravlić, Zlatko Voloder, Feri Čiradžija, Krunomir Dvorski, Branko Kuzmanović and Eytan Suchard. Mrs. Jasna Gašparović and Miss Mia Perkovac kindly provide me language support. Thanks to my wife and my family.

\section{References}

[1] Cole J. M., Behm K.T., Blackburn T. G., Wood J. C., Baird C. D., Duff M. J., Harvey C., Ilderton A., Joglekar A. S. , Krushelnik K., Kuschel S., Marklund M., McKenna P., Murphy C. D., Poder K., Ridgers C. P., Samarin G. M., Sarri G., Symes D. R., Thomas A. G. R., Warwick J., Zepf M., Najmudin Z. and Mangles S. P. D. (2018). Experimental evidence of radiation reaction in the collision of a high-intensity laser pulse with a laser-wakefield accelerated electron beam. arXiv:1707.06821v2, 1 .

[2] Schpolski E. W. (1979). Atomic physics I (Atomphysik I). 15th Newly Revised Edition, VEB Deutscher Verlag der Wissenschaften, Berlin.

[3] Supek I. (1974). Theoretical physics and structure of matter I (Teorijska fizika i struktura materije I), 4th Edition, Školskaknjiga, Zagreb.

[4] Planck M. (1944). Paths to physical knowledge (Wege zur Physikalische Erkenntnis), 4th Edition, Verlag von S. Hirzel in Leipzig.

[5] Perkovac M. (2002). Quantization in Classical Electrodynamics. Physics Essays, 15, 41-60.

[6] Perkovac M. (2003). Absorption and Emission of Radiation by an Atomic Oscillator. Physics Essays, 16, 162-173.

[7] Jackson J. D. (1998). Classical Electrodynamics, 3rd Edition, John Wiley \& Sons, Inc, New York, Chichester, Weinheim, Brisbane, Singapore, Toronto.

[8] Giancoli D. C. (1988). Physics for scientists and engineers, 2nd Edition, Prentice-Hall, London, Sydney, Toronto, Mexico, New Delhi, Tokyo, Simon \& Schuster Asia, Pte. Ltd., Singapore, Editora Prentice-Hall do Brasil, Rio de Janeiro.

[9] NIST database. (2020). NIST Atomic Spectra Database Ionization Energies Form. https://physics.nist.gov/PhysRefData/ASD/ion Energy.html.

[10] Kurnik Z. (2007). Diophantine Equations (Diofantske jednadžbe), Hrvatsko matematičko društvo, Zagreb.

[11] Surutka J. (1971). Electromagnetics (Elektromagnetika), 3rd Edition, Građevins kaknjiga, Beograd. 
[12] Perkovac M. (2014). Maxwell's Equations as the Basis for Model of Atoms, Journal of Applied Mathematics and Physics, 2, 235-251.

[13] Perkovac M. (2010). Statistical test of Duane-Hunt's law and its comparison with an alternative law.arXiv:1010.6083, 2.

[14] Duane W. and Hunt F. L. (1915). On X-Ray Wave-Lengths, Phys. Rev. 6, 166-172.

[15] Hänsel H., Neumann W. (1995). Physics; Atoms-Atomic nuclei-Elementary particles (Physik; Atome-Atomkerne-Elementarteilchen). Spektrum Akademischer Verlag, Heidelberg • Berlin• Oxford.

[16] Perkovac M. (2013). Model of an Atom by Analogy with the Transmission Line. Journal of Modern Physics, 4, 899-903.

[17] Perkovac M. (2014). Determination of the Structural Constant of the Atom. Journal of Applied Mathematics and Physics, 2, 11-21.

[18] Haznadar Z., Štih Ž. (1997). Electromagnetism (Elektromagnetizam), Školska knjiga, Zagreb.

[19] Kulenkampff H. (1926). Continuous X-ray Spectrum (Das Kontinuirliche Röntgenspektrum), In: Bothe W. at. al., Ed., Quanten. Handbuch der Physik, vol. 23, Springer, Berlin, Heidelberg, 433-476.

[20] Perkovac M. (2018). Measurement of Type of Substance Based on Protons, Neutrons and Electrons in the Substance. Asian Journal of Physical and Chemical Sciences, 4(4), 1-8. 